\title{
Multi-Attribute Decision-Making Based on Prioritized Aggregation Operator under Hesitant Intuitionistic Fuzzy Linguistic Environment
}

\author{
Peide Liu ${ }^{1, *}\left(\mathbb{D}\right.$, Tahir Mahmood ${ }^{2}$ and Qaisar Khan ${ }^{2}$ \\ 1 School of Management Science and Engineering, Shandong University of Finance and Economics, \\ Jinan 250014, China \\ 2 Department of Mathematics and Statistics, International Islamic University, Islamabad 44000, Pakistan; \\ tahirbakhat@yahoo.com (T.M.); qaisarkhan421@gmail.com (O.K.) \\ * Correspondence: Peide.liu@gmail.com
}

Academic Editor: Hari Mohan Srivastava

Received: 26 October 2017; Accepted: 5 November 2017; Published: 9 November 2017

\begin{abstract}
A hesitant intuitionistic fuzzy linguistic set (HIFLS) that integrates both qualitative and quantitative evaluations is an extension of the linguistic set, intuitionistic fuzzy set (IFS), hesitant fuzzy set (HFS) and hesitant intuitionistic fuzzy set (HIFS). It can describe the qualitative evaluation information given by the decision-makers (DMs) and reflect their uncertainty. In this article, we defined some new operational laws and comparative method for HIFLSs. Then, based on these operations, we propose two prioritized aggregation (PA) operators for HIFLSs: prioritized weighted averaging operator for HIFLSs (HIFLPWA) and prioritized weighted geometric operator for HIFLSs (HIFLPWG). Based on these aggregation operators, an approach for multi-attribute decision-making (MADM) is developed under the environment of HIFLSs. Finally, a practical example is given to show the practicality and effectiveness of the developed approach by comparing with the other representative methods.
\end{abstract}

Keywords: linguistic set; hesitant intuitionistic fuzzy numbers; aggregation operators; linguistic scale function; multi-attribute decision-making (MADM)

\section{Introduction}

In modern decision science, one of the most important research topics is multi-attribute decision-making (MADM) which can select the best alternative according to multiple influential attributes [1-3] Generally, the decision-makers (DMs) use crisp numbers to manifest their preferences about the alternative in traditional MADM problems. However, due to deficiency of information, insufficiency of data and time pressure, the attribute values, especially for qualitative attribute values, generally cannot be manifested by real numbers, and some of them are easier to be expressed by fuzzy values. Since Zadeh [4] presented fuzzy set (FS), many extensions of FSs were made by researchers [5-7]. In Zadeh' FSs, there is only the membership degree (MD) with a single value in the closed interval $[0,1]$. Obviously, it is insufficient to depict complete information due to lack of comprehensive and systematic knowledge.

To deal with such cases, Torra [8] made an extension of the FSs and proposed the concept of hesitant fuzzy set (HFS). In HFSs, the MD of an element in a universe is a set of several possible values in the closed interval [0, 1]. After that, many MADM methods [9-14] and measures including correlation, distance, similarity and entropy [15-18] have been proposed for HFS by many researchers. Liao and $\mathrm{Xu}[19,20]$ presented the subtraction and division operations, and hybrid arithmetical averaging for HFSs (HFHAA), hybrid arithmetical geometric for HFSs (HFHAG), the quasi HFHAA and the quasi HFHAG operators, $\mathrm{Xia}$ and $\mathrm{Xu}$ [21], and Zhang [22] proposed power aggregation operators for 
HFS and successfully they are applied to MADM. Zhu et al. [23] presented the dual HFSs (DHFSs). Singh [24] proposed some distance and similarity measures for DHFSs.

When the problems faced that are too ill-defined or complex are described by quantitative expression, the linguistic variables (LVs) are an efficient tool to depict quantitative information [25]. LVs have been studied and applied by many researchers in various fields [2,26-29], and may be a single linguistic term (LT) [30] or interval LTs, namely uncertain LVs. Rodriguez et al. [31,32] presented the hesitant fuzzy LT set which contains several LTs. Moreover, by combining LVs and HFSs, Lin et al. [33] introduced the hesitant fuzzy linguistic set (HFLS), in which the LV is with a set of several possible MDs in the closed interval [0,1]. After that, Wu et al. [34] found some limitations in the HFLS and proposed some new operations based on the linguistic scale function (LSF). However, in HFLSs, only the MD was considered and the non-membership degree (NMD) is ignored, which may cause information distortion and loss. IFS introduced by Atanassov [5] consists of MD and NMD, i.e., the MD is explained as the epistemic certainty, while the NMD is explained as the epistemic negativity. IFSs have been widely used by many researchers and participants $[35,36]$. Motivated by the idea of IFS and HFLS, Liu et al. [37] presented hesitant intuitionistic fuzzy linguistic set (HIFLS), hesitant intuitionistic fuzzy linguistic element (HIFLE) and defined some basic operational rules, score and accuracy functions for HIFLEs. Then, based on these operational rules, some aggregation operators were defined and applied to MADM. However, there are some existing limitations in the operations, the score and accuracy functions defined for HIFLEs.

Aggregation operators are necessary tools in information fusion in MADM problems [38-41]. In practical situation, generally, DMs should consider prioritization between different criteria. To handle such kind of situation, Yager [21] was the first to propose PA operator and applies it to MADM problems. Liu et al. [42] proposed prioritized OWA operator to deal with intuitionistic trapezoidal fuzzy information. However, in [21], the criteria values were discussed in real number domain, and there have been no aggregation operators that consider the priorities for HIFLNs.

The advantage of the HIFLEs is they can fully depict complex uncertain information; however, there are some shortcomings in the operational rules and the comparison method of HIFLEs, for example, when one NMD of HIFLEs equals to zero, then the effects of other NMDs on operational result of addition operation do not play a significant role. In addition, some HIFLEs cannot be compared by the existing methods. Thus, it is necessary to study the new operational rules and comparison method. Moreover, the PA operator has the advantage that it can consider the priority among the criteria, and it can solve the MADM problems with priority among the attributes. Therefore, the purpose of this article is: (1) to propose new operational results for HIFLEs to overcome the existing shortcomings; (2) to develop a new comparison method for HIFLEs; (3) to propose some PA operators for HIFLEs; and (4) to develop a decision method for the MADM problems with priority among the attributes based on the proposed operators.

The rest of the article is organized as follows. In Section 2, some basic concepts about LT sets (LTSs), HFSs, HFLSs, IFS, LSF and PA operators are briefly reviewed. In Section 3, we explain some existing limitations in the operations, score and accuracy function of HIFLEs, and define some new operations, score and accuracy functions for HIFLEs. In Section 4, two prioritized aggregation operators for HIFLEs are developed. In Section 5, based on these defined operators, a MCDM method is proposed. In Section 6, comparison and discussion with the existing aggregation operators through the same example are illustrated. Finally, conclusion and references are given.

\section{Preliminaries}

\subsection{LTS}

Let $S=\left\{s_{0}, s_{2}, \ldots, s_{z}\right\}$ be a finite LTS with odd cardinality, where $s_{j}$ is a possible value from the LT, and $z+1$ is the length of $S$. For example, when $z=4$, a set of five LTs $S$ is defined as follows:

$$
S=\left\{s_{0}=\text { verylow, } s_{1}=\text { low, } s_{2}=\text { medium }, s_{3}=\text { high }, s_{4}=\text { very high }\right\}
$$


Clearly, the middle LT denotes the assessments of "indifference" and the other LTs are set on both sides of it symmetrically. In such cases, for any LTS $S, s_{u}$ and $s_{v}$ can satisfy the following properties:

(1) The set is ordered: $s_{u}>s_{v}$, if $u>v$;

(2) Negation operator: $\operatorname{Neg}\left(s_{u}\right)=s_{v}$ such that $v=z-u$;

(3) Maximum operator: $\max \left(s_{u}, s_{v}\right)=s_{u}$, if $u \geq v$; and

(4) Minimum operator: $\min \left(s_{u}, s_{v}\right)=s_{u}$, if $u \leq v$.

To keep the information, the discrete LTS $S$ is extended to a continuous LTS (CLTS) $\bar{S}=\left\{s_{\vartheta} \mid \vartheta \in[0, z]\right\}$ which satisfies all the properties defined above. If $s_{\vartheta} \in S$, then $s_{\vartheta}$ is an original LT. Otherwise, $s_{\vartheta}$ is the virtual LT. Generally, the DMs can use original LT for the assessment of alternatives and the virtual LT is only used in the calculation [2]. The algebraic operational laws of the LVs can be found in $[2,3]$.

\subsection{HFLS}

Definition 1 [8]. Let $U$ be a non-empty fixed set, then a HFS on $U$ can be defined as

$$
H=\{\langle u, h(u)\rangle \mid u \in u\}
$$

where $h(u)=\cup_{v(u) \in h(u)}\{v(u)\}$ is a group of some values in $[0,1]$ expressing the possible MDs of the element $u \in U$ to the set $H$, the $h(u)$ is called a hesitant fuzzy element (HFE), and $H$ is regarded as the set of all HFEs [43].

Definition 2 [43]. Let $h_{1}=\cup_{v_{1} \in h_{1}}\left\{v_{1}\right\}, h_{2}=\cup_{v_{2} \in h_{2}}\left\{v_{2}\right\}$ be two HFEs, and $\kappa \geq 0$, then some operational laws for HFEs are given as follows:

$$
\begin{gathered}
h_{1}+h_{2}=\cup_{v_{1} \in h_{1}, v_{2} \in h_{2}}\left\{1-\left(1-v_{1}\right)\left(1-v_{2}\right)\right\} ; \\
h_{1} \times h_{2}=\cup_{v_{1} \in h_{1}, v_{2} \in h_{2}}\left\{v_{1} v_{2}\right\} ; \\
\kappa h_{1}=\cup_{v_{1} \in h_{1}}\left\{1-\left(1-v_{1}\right)^{\kappa}\right\} \\
h_{1}^{\kappa}=\cup_{v_{1} \in h_{1}}\left\{v_{1}{ }^{\kappa}\right\} .
\end{gathered}
$$

Definition 3 [33]. Let $U$ be a non-empty universal set and $\bar{S}$ be a CLTS of $S=\left\{s_{0}, s_{1}, \ldots, s_{z}\right\}$, then a HFLS on $U$ can be represented by

$$
\widetilde{D}=\left\{\left\langle u, s_{\vartheta(u)}, h_{\widetilde{D}}(u)\right\rangle \mid u \in U\right\}
$$

where $s_{\vartheta(u)} \in \bar{S}, h_{\widetilde{D}}(u)=\cup_{v_{\widetilde{D}}(u) \in h_{\widetilde{D}}(u)}\left\{v_{\widetilde{D}}(u)\right\}$ is a group of some possible MDs of the element $u \in U$ to the $L T s_{\vartheta(u)}$ in $[0,1]$. Then, $\widetilde{d}=\left\langle u, s_{\vartheta(u)}, h_{\widetilde{D}}(u)\right\rangle$ is called hesitant fuzzy linguistic number (HFLN).

\subsection{IFS}

Definition 4 [5]. Let $U$ be a universal set. An IFS $M$ on $U$ is defined as follows:

$$
M=\{\langle a, m(a), n(a)\rangle \mid a \in U\}
$$

where $m(a)$ and $n(a)$, respectively, represent the $M D$ and NMD of the element $a \in U$ to the set $M$. Then, $\widetilde{m}=\langle m, n\rangle$ is called an intuitionistic fuzzy number (IFN).

Definition 5 [5]. Let $\widetilde{m}_{1}=\left\langle m_{1}, n_{1}\right\rangle$ and $\widetilde{m}_{2}=\left\langle m_{2}, n_{2}\right\rangle$ be two IFNs and $\kappa \geq 0$. Then, some basic operational rules for IFNs are defined below:

(1) $\widetilde{m}_{1}+\widetilde{m}_{2}=\left\langle m_{1}+m_{2}-m_{1} m_{2}, n_{1} n_{2}\right\rangle$; 
(2) $\widetilde{m}_{1} \times \widetilde{m}_{2}=\left\langle m_{1} m_{2}, n_{1}+n_{2}-n_{1} n_{2}\right\rangle$;

(3) $\widetilde{m}_{1}^{\kappa}=\left\langle m_{1}^{\kappa}, 1-\left(1-n_{1}\right)^{\kappa}\right\rangle ;$ and

(4) $\kappa \widetilde{m}_{1}=\left\langle 1-\left(1-m_{1}^{\kappa}\right), n_{1}{ }^{\kappa}\right\rangle$.

\subsection{PA Operators}

Definition 6 [21]. Let $\widetilde{G}=\left\{\widetilde{G}_{1}, \widetilde{G}_{2}, \ldots, \widetilde{G}_{l}\right\}$ be a family of attributes, and ensure that there exist a prioritization among the attributes represented by a linear ordering $\widetilde{G}_{1}>\widetilde{G}_{2}>\ldots>\widetilde{G}_{l}$, which denote that the criterion $\widetilde{G}_{d}$ has a high priority than $\widetilde{G}_{f}$, if $d<f$. $\widetilde{G}_{d}(u)$ is an assessment value representing the performance of the alternative $u$ under the criterion $\widetilde{G}_{d}$ and satisfies $\widetilde{G}_{d} \in[0,1]$. if

$$
P A\left(\widetilde{G}_{1}(u), \widetilde{G}_{2}(u), \cdots, \widetilde{G}_{l}(u)\right)=\sum_{d=1}^{l} w_{d} \widetilde{G}_{d}(u)
$$

then PA operator has been successfully applied in a situation where the attributes are real values. Therefore, the PA operator can be extended to the HIFLNs.

\section{5. $L S F$}

To describe the semantics more flexibly, LSFs are assigned to different semantic values based on the different application situations [44].

Definition 7 [44]. Let $s_{j} \in S$ be a $L T$, where $S=\left\{s_{j} \mid j=0,1,2, \ldots, 2 k\right\}$. If $\theta_{j} \in[0,1]$ is a numerical value, then the LSF $F^{*}$ is a function from $s_{j}$ to $\theta_{j}(j=0,1,2, \ldots, 2 k)$, and is defined as follows:

$$
F^{*}: s_{j} \rightarrow \theta_{j}(j=0,1, \ldots, 2 k)
$$

where $0 \leq \theta_{0}<\theta_{1}<\ldots<\theta_{2 k}$. Clearly, the symbol $\theta_{j}(j=0,1,2, \ldots, 2 k)$ reflects the preference of DMs when they are using the $L T s_{j} \in S(j=0,1,2, \ldots, 2 k)$. Thus, the function value can express the semantics of the LTs. There are three types of LSFs.

(1) The first type of LSF is defined as:

$$
F_{1}^{*}\left(s_{j}\right)=\theta_{j}=\frac{j}{2 k}(j=0,1, \ldots, 2 k)
$$

Here $\theta_{j} \in[0,1]$, and all LTs are divided evenly in [0, 1].

(2) The second type of LSF is defined as:

$$
F_{2}^{*}\left(s_{j}\right)=\theta_{j}=\left\{\begin{array}{lr}
\frac{\gamma^{k}-\gamma^{k-j}}{2 \gamma^{k}-2} & (j=0,1,2, \ldots, k) \\
\frac{\gamma^{k}+\gamma^{j-k}-2}{2 \gamma^{k}-2} & (j=k+1, k+2 \ldots, 2 k)
\end{array}\right.
$$

The value of $\gamma$ can be determined by $\gamma=\sqrt[k]{m}$ [44], where $k$ represents the scale level, and $m$ is the importance ratio, of which indicator A is far more important than indicator B. In general, $m=9$. If $k=7$, then $\gamma=\sqrt[7]{9}=1.37$.

Then, all LTs are non-uniformly distributed in $[0,1]$, and the closer to both ends the LT is, the more deviation there is between adjacent two LTs.

(3) The third type of LSF is defined as

$$
F_{3}^{*}\left(s_{j}\right)=\theta_{j}=\left\{\begin{array}{lr}
\frac{k^{\alpha}-(k-j)^{\alpha}}{2 k^{\alpha}} & (j=0,1,2, \ldots, k) \\
\frac{k^{\beta}-(j-k)^{\beta}}{2 k^{\beta}} & (j=k+1, k+2 \ldots, 2 k)
\end{array}\right.
$$


Here $\alpha, \beta \in(0,1]$. If $\alpha=\beta=1$, then $\theta_{j}=\frac{j}{2 k}$. In this situation, all LTs are non-uniformly distributed in $[0,1]$, and the closer to both ends the LT is, the smaller the deviation is between two adjacent LTs.

To keep all the given information, the above-mentioned functions can be extended to $F^{*}: \bar{S} \rightarrow \theta(\theta \in[0,1])$, where $F^{*}$ is a strictly monotonically increasing and continuous function. Then, the inverse function of $F^{*}$ is expressed by $F^{*-1}$.

\section{HIFS and Their Operations}

\subsection{The Definition and Operational Rules of HIFLNs}

Definition 8 [37]. Let $U$ be the universe of discourse set and $\bar{S}$ be a CLTS. Then a HIFLS is an object which is mathematically represented as

$$
A=\left\{\left\langle u, s_{\vartheta(u)}, h_{A}(u)\right\rangle \mid u \in U\right\}
$$

where $s_{\vartheta(u)} \in \bar{S}, h_{A}(u)=\underset{\left(m_{A}(u), n_{A}(u)\right) \in h_{A}(u)}{\cup}\left\{m_{A}(u), n_{A}(u)\right\}$ is a set of IFNs, respectively representing the some possible MDs and NMDs of the element $u \in U$ to the $L T s_{\vartheta(u)}$. In addition, we shall call $a=\left\langle s_{\vartheta(u)}, h_{A}(u)\right\rangle a$ HIFLN.

Definition 9 [37]. Let $a_{1}=\left\langle s_{\vartheta\left(a_{1}\right)}, h\left(a_{1}\right)\right\rangle$ and $a_{2}=\left\langle s_{\vartheta\left(a_{2}\right)}, h\left(a_{2}\right)\right\rangle$ be any two HIFLNs and $\rho \geq 0$. Then, some operational laws for HIFLNs are defined as follows:

$$
\begin{gathered}
a_{1}+a_{2}=\left\langle s_{\vartheta\left(a_{1}\right)+\vartheta\left(a_{2}\right),}{ }_{\left(m\left(a_{1}\right), n\left(a_{1}\right)\right) \in h\left(a_{1}\right),\left(m\left(a_{2}\right), n\left(a_{2}\right)\right) \in h\left(a_{2}\right)}^{\cup}\left\{m\left(a_{1}\right)+m\left(a_{2}\right)-m\left(a_{1}\right) m\left(a_{2}\right), n\left(a_{1}\right) n\left(a_{2}\right)\right\}\right\rangle ; \\
a_{1} \times a_{2}=\left\langle s_{\vartheta\left(a_{1}\right) \times \vartheta\left(a_{2}\right),}\right. \\
\rho a_{1}=\left\langle s_{\rho \times \vartheta\left(a_{1}\right),} \underset{\left.\left(m\left(a_{1}\right), n\left(a_{1}\right)\right) \in h\left(a_{1}\right),\left(m\left(a_{2}\right), n\left(a_{2}\right)\right) \in h\left(a_{1}\right)\right) \in h\left(a_{1}\right)}{\cup}\left\{m\left(a_{1}\right) m\left(a_{2}\right), n\left(a_{1}\right)+n\left(a_{2}\right)-n\left(a_{1}\right) n\left(a_{2}\right)\right\}\right\rangle ; \\
\rho a_{1}=\left\langle s_{\vartheta \rho\left(a_{1}\right)}, \underset{\left(m\left(a_{1}\right), n\left(a_{1}\right)\right) \in h\left(a_{1}\right)}{\cup}\left\{\left(m\left(a_{1}\right)\right)^{\rho}, 1-\left(1-n\left(a_{1}\right)\right)^{\rho}\right\}\right\rangle .
\end{gathered}
$$

Obviously, the above operations for HIFLNs defined by Liu et al. [37] have some limitations, which are stated as follows.

(1) In the additive operation in Equation (14), if only one NMD equals zero, then the effects of other grades on the overall aggregated result do not play a significant role in the aggregation process.

(2) In the additive operation in Equation (14), the two parts including LVs and IFNs of HIFLNs are completely independent in operations, which may disregard the correlation among them. Take $a=\left\langle s_{3},\{0.2,0.3\},\{0.4,0.5\}\right\rangle$; for example, $\{0.2,0.3\}$ and $\{0.4,0.5\}$ are IFNs representing the possible MDs and NMDs of the element that belongs to $s_{3}$; that is, $\{0.2,0.3\}$ and $\{0.4,0.5\}$ are the explanatory parts of $s_{3}$ and should be closely related in the additive operations.

(3) The negation operator for HIFLN is not defined.

To solve the above shortcomings, we define some new operational rules for HIFLNs based on LSFs as follows. 
Definition 10. Let $a_{1}=\left\langle s_{\vartheta\left(a_{1}\right)}, h\left(a_{1}\right)\right\rangle$ and $a_{2}=\left\langle s_{\vartheta\left(a_{2}\right)}, h\left(a_{2}\right)\right\rangle$ be any two HIFLNs and $\rho \geq 0$. Then, some new operational laws for HIFLNs are given as follows:

$$
\begin{aligned}
& a_{1}+a_{2}=\left\{\begin{array}{c}
F^{*-1}\left(F^{*}\left(s_{\vartheta\left(a_{1}\right)}\right)+F^{*}\left(s_{\vartheta\left(a_{2}\right)}\right)\right), \\
\left\{\begin{array}{c}
\left.\cup\left(a_{1}\right), n\left(a_{1}\right)\right) \in h\left(a_{1}\right),\left(m\left(a_{2}\right), n\left(a_{2}\right)\right) \in h\left(a_{2}\right) \\
\left.\frac{F^{*}\left(s_{\vartheta\left(a_{1}\right)}\right) m\left(a_{1}\right)+F^{*}\left(s_{\vartheta\left(a_{2}\right)}\right) m\left(a_{2}\right)}{F^{*}\left(s_{\vartheta\left(a_{1}\right)}\right)+F^{*}\left(s_{\vartheta\left(a_{2}\right)}\right)}, \frac{F^{*}\left(s_{\vartheta\left(a_{1}\right)}\right) n\left(a_{1}\right)+F^{*}\left(s_{\vartheta\left(a_{2}\right)}\right) n\left(a_{2}\right)}{F^{*}\left(s_{\vartheta\left(a_{1}\right)}\right)+F^{*}\left(s_{\vartheta\left(a_{2}\right)}\right)}\right\}
\end{array}\right\} ;
\end{array}\right. \\
& a_{1} \times a_{2}=\left\langle\begin{array}{c}
F^{*-1}\left(F^{*}\left(s_{\vartheta\left(a_{1}\right)}\right)+F^{*}\left(s_{\vartheta\left(a_{2}\right)}\right)\right), \\
\left.\left\{\left\{m\left(a_{1}\right) m\left(a_{2}\right), n\left(a_{1}\right)+n\left(a_{1}\right), n\left(a_{1}\right)\right) \in h\left(a_{1}\right),\left(m\left(a_{2}\right), n\left(a_{2}\right)\right) \in h\left(a_{2}\right) n\left(a_{2}\right)\right\}\right\}
\end{array}\right\rangle ; \\
& \rho a_{1}=\left\langle F^{*-1}\left(\rho F^{*}\left(s_{(\vartheta)}\right), h(a)\right\rangle ;\right. \\
& a_{1}^{\rho}=\left\langle F^{*-1}\left(\left(F^{*}\left(s_{(\vartheta)}\right)\right)^{\rho}\right), \underset{\left(m\left(a_{1}\right), n\left(a_{1}\right)\right) \in h\left(a_{1}\right)}{\cup}\left\{\left(m\left(a_{1}\right)\right)^{\rho}, 1-\left(1-n\left(a_{1}\right)\right)^{\rho}\right\}\right\rangle ; \\
& n e g a_{1}=\left\langle F^{*-1}\left(\left(F^{*}\left(s_{2 t}\right)-F^{*}\left(s_{\vartheta\left(a_{1}\right)}\right)\right)\right), \underset{\left(m\left(a_{1}\right), n\left(a_{1}\right)\right) \in h\left(a_{1}\right)}{\cup}\left\{n\left(a_{1}\right), m\left(a_{1}\right)\right\}\right\rangle .
\end{aligned}
$$

Example 1. Let $S=\left\{s_{0}, s_{1}, s_{2}, s_{3}, s_{4}, s_{5}, s_{6}\right\}=$ \{very poor, slightly poor, poor, fair, slightly good, good, very good $\}, a_{1}=\left\langle s_{1},\{(0.2,0.6),(0.4,0.5)\}\right\rangle, a_{2}=\left\langle s_{4},\{(0.5,0.2),(0.6,0.1)\}\right\rangle$ and $\rho=3$,

(1) If $F_{1}^{*}\left(s_{i}\right)=\frac{i}{6}(0 \leq i \leq 6)$, then

$$
\begin{gathered}
a_{1}+a_{2}=\left\langle s_{5.0004},\{(0.4400,0.2800),(0.52,0.20),(0.48,0.26),(0.56,0.15)\}\right\rangle ; \\
a_{1} \times a_{2}=\left\langle s_{0.6668},\{(0.1,0.68),(0.12,0.64),(0.2,0.6),(0.24,0.55)\}\right\rangle ; \\
3 a_{1}=\left\langle s_{3},\{(0.2,0.6),(0.4,0.5)\}\right\rangle ; \\
a_{1}^{3}=\left\langle s_{0.0278},\{(0.008,0.936),(0.064,0.875)\}\right\rangle ; \\
\text { neg } a_{1}=\left\langle s_{5},\{(0.6,0.2),(0.5,0.4)\}\right\rangle .
\end{gathered}
$$

(2) If $F_{2}^{*}\left(s_{i}\right)=\left\{\begin{array}{cc}\frac{\kappa^{r}-\kappa^{r-i}}{2 \kappa^{r}-2} & (0 \leq i \leq r) \\ \frac{\kappa^{r}+\kappa^{i-r}-2}{2 \kappa^{r}-2} & (r \leq i \leq 2 r)\end{array}, \kappa=1.4\right.$, then

$$
\begin{gathered}
a_{1}+a_{2}=\left\langle s_{5.322,},\{(0.4197,0.3607),(0.4929,0.2339),(0.4732,0.2803),(0.5464,0.2071)\}\right\rangle ; \\
a_{1} \times a_{2}=\left\langle s_{0.5741},\{(0.1,0.68),(0.12,0.64),(0.2,0.6),(0.24,0.55)\}\right\rangle ; \\
3 a_{1}=\left\langle s_{4.41},\{(0.2,0.6),(0.4,0.5)\}\right\rangle ; \\
a_{1}^{3}=\left\langle s_{0.0433},\{(0.008,0.936),(0.064,0.875)\}\right\rangle ; \\
n \operatorname{neg} a_{1}=\left\langle s_{5},\{(0.6,0.2),(0.5,0.4)\}\right\rangle .
\end{gathered}
$$

(3) If $F_{3}^{*}\left(s_{i}\right)=\left\{\begin{array}{cc}\frac{r^{\zeta}-(r-i)^{\zeta}}{2 r^{\zeta}} & (0 \leq i \leq r) \\ \frac{r^{\eta}+(i-r)^{\eta}}{2 r^{\eta}} & (r \leq i \leq 2 r)\end{array}, \zeta=\eta=0.8\right.$, then

$$
\begin{gathered}
a_{1}+a_{2}=\left\langle s_{4.894,},\{(0.451,0.2655),(0.5345,0.1539),(0.4836,0.2491),(0.5672,0.1654)\}\right\rangle ; \\
a_{1} \times a_{2}=\left\langle s_{0.7164},\{(0.1,0.68),(0.12,0.64),(0.2,0.6),(0.24,0.55)\}\right\rangle ; \\
3 a_{1}=\left\langle s_{2.675},\{(0.2,0.6),(0.4,0.5)\}\right\rangle ;
\end{gathered}
$$




$$
\begin{gathered}
a_{1}^{3}=\left\langle s_{0.0199},\{(0.008,0.936),(0.064,0.875)\}\right\rangle ; \\
n e g a_{1}=\left\langle s_{5},\{(0.6,0.2),(0.5,0.4)\}\right\rangle .
\end{gathered}
$$

\subsection{Comparison Method for HIFLNs}

The comparison method for HIFLNs defined by Liu et al. [37] is given below.

Definition 11 [37]. Suppose $a=\left\langle s_{\vartheta(a)}, h(a)\right\rangle$ is a HIFLN. Then, the score function (SF) E(a) is defined as follows:

$$
E(a)=\left(\frac{1}{\neq h(a)} \sum_{(m(a), n(a)) \in h(a)}(m(a)-n(a))\right) \frac{\vartheta(a)}{r}
$$

where $\neq h(a)$ is the number of IFNs in $h(a)$, and $r+1$ is the cardinality of the LTS S. The greater is the SF $E(a)$, the greater is the HIFLN $a$.

Definition 12 [37]. Suppose $a=\left\langle s_{\vartheta(a)}, h(a)\right\rangle$ is a HIFLN. Then the accuracy function $(A F) H(a)$ is defined as follows:

$$
H(a)=\left(\frac{1}{\neq h(a)} \sum_{(m(a), n(a)) \in h(a)}(m(a)+n(a))\right) \frac{\vartheta(a)}{r}
$$

where $\neq h(a)$ is the number of IFNs in $h(a)$, and $r+1$ is the cardinality of the LTS $S$. The greater is the AF $H(a)$, the greater is the HIFLN $a$.

Based on the above SF and AF, the comparison rules given by Liu et al. [37] show the following:

Definition 13 [37]. Let $a_{1}=\left\langle s_{\vartheta\left(a_{1}\right)}, h\left(a_{1}\right)\right\rangle$ and $a_{2}=\left\langle s_{\vartheta\left(a_{2}\right)}, h\left(a_{2}\right)\right\rangle$ be two HIFLNs, and $E\left(a_{i}\right)$ and $H\left(a_{i}\right)$ are, respectively, the SFs and AFs $a_{i}(i=1,2)$, then

(1) If $E\left(a_{1}\right)<E\left(a_{2}\right)$, then $a_{1}<a_{2}$; and

(2) If $E\left(a_{1}\right)=E\left(a_{2}\right)$, then

(a) If $H\left(a_{1}\right)<H\left(a_{2}\right)$, then $a_{1}<a_{2}$; and

(b) If $H\left(a_{1}\right)=H\left(a_{2}\right)$, then $a_{1}=a_{2}$.

The SF and AF defined by Liu et al. [37] have some limitations in some special cases for comparing two HIFLNs; they can be shown by an example.

Example 2. Let $S=\left\{s_{0}, s_{1}, s_{2}, s_{3}, s_{4}, s_{5}, s_{6}\right\}, a_{1}=\left\langle s_{4},\{(0.7,0.3),(0.8,0.2)\}\right\rangle$ and

$$
a_{2}=\left\langle s_{4},\{(0.9,0.1),(0.6,0.4)\}\right\rangle .
$$

Then, by the above SF and AF defined by Liu et al. [37], we have,

$$
E\left(a_{1}\right)=E\left(a_{2}\right)=0.750, H\left(a_{1}\right)=H\left(a_{2}\right)=0.750 .
$$

Obviously, these two HIFLNs $a_{1}$ and $a_{2}$ cannot be compared. To overcome the above existing limitation in this example, we define new SF and AF based on LSFs for comparing with HIFLNs.

Definition 14. Let $a=\left\langle s_{\vartheta(a)}, h(a)\right\rangle$ be a HIFLN, and then the SF S $(a)$ can be defined as follows:

$$
S(a)=F^{*}\left(s_{\vartheta(a)}\right) \times C(h(a))
$$


where $C(h(a))=\frac{1}{\neq h} \sum_{(m(a), n(a)) \in h(a)}\left(\frac{(m(a))+1-n(a)}{2}\right) . \neq h$ represents the number of IFNs in $h(a)$.

Definition 15. Let $a=\left\langle s_{\vartheta}(a), h(a)\right\rangle$ be a HIFLN. A variance function $D(h(a))$ of $h(a)$ is represented as $D(h(a))=\frac{1}{\neq h} \sum_{(m(a), n(a)) \in h(a)}\left(\left(\frac{(m(a))+1-n(a)}{2}\right)-C(h(a))\right)^{2}$. Thus, the AF $A(h(a))$ is given as follows:

$$
A(a)=F^{*}\left(s_{\vartheta(a)}\right) \times[1-D(h(a))]
$$

where $\neq h$ represents the number of IFNs in $h(a)$.

Definition 16. Let $a_{1}=\left\langle s_{\vartheta\left(a_{1}\right)}, h\left(a_{1}\right)\right\rangle$ and $a_{2}=\left\langle s_{\vartheta\left(a_{2}\right)}, h\left(a_{2}\right)\right\rangle$ be two HIFLNs, and let $E\left(a_{i}\right)$ and $H\left(a_{i}\right)$ be, respectively, the SFs and AFs of $a_{i}(i=1,2)$, then

(1) If $S\left(a_{1}\right)<S\left(a_{2}\right),\left(S\left(a_{1}\right)>S\left(a_{2}\right)\right)$, then $a_{1}<a_{2}\left(a_{1}>a_{2}\right)$; and

(2) If $S\left(a_{1}\right)=S\left(a_{2}\right)$, then

(a) If $A\left(a_{1}\right)<A\left(a_{2}\right),\left(A\left(a_{1}\right)>A\left(a_{2}\right)\right)$, then $a_{1}<a_{2},\left(a_{1}>a_{2}\right)$; and

(b) If $A\left(a_{1}\right)=A\left(a_{2}\right)$, then $a_{1}=a_{2}$.

Example 3. Consider Example 2 by Definition 16, and we take $F^{*}\left(s_{\vartheta}\right)=\frac{i}{2 r}$, then,

$$
\begin{gathered}
S\left(a_{1}\right)=0.5, S\left(a_{2}\right)=0.5 \\
A\left(a_{1}\right)=0.6650, A\left(a_{2}\right)=0.6517 .
\end{gathered}
$$

Thus, we have $a_{1}>a_{2}$.

\section{Prioritized Aggregation Operators for HIFLNs}

In this part, we propose some PA operators based on new operational laws for HIFLNs.

\subsection{The HIFLPWA Operator}

Definition 17. Let $a_{j}(j=1,2, \ldots, k)$ be a group of HIFLNs, then the HIFLPWA operator is given as follows:

$$
\operatorname{HIFLPWA}\left(a_{1}, a_{2}, \ldots, a_{n}\right)=\frac{T_{1}}{\sum_{z=1}^{k} T_{z}} a_{1}+\frac{T_{2}}{\sum_{z=1}^{k} T_{z}} a_{2}+\ldots+\frac{T_{k}}{\sum_{z=1}^{k} T_{z}} a_{k}=\oplus_{j=1}^{k} \frac{T_{j} a_{j}}{\sum_{z=1}^{k} T_{z}}
$$

where $T_{j}=\prod_{l=1}^{j-1} S\left(a_{l}\right)(j=2,3, \ldots, k), T_{1}=1$, and $S\left(a_{l}\right)$ is the SF of $a_{l}$.

Based on new operational laws defined for HIFLNs, we have the following theorem.

Theorem 1. Suppose $a_{j}(j=1,2, \ldots, k)$ is a group of HIFLNs. Then, the aggregated result obtained by Definition 17 is also a HIFLN, and

$$
\begin{aligned}
\operatorname{HIFLPWA}\left(a_{1}, a_{2}, \ldots, a_{k}\right)= & \left\langle F^{*-1}\left(\sum_{j=1}^{k}\left(\frac{T_{j}}{\sum_{z=1}^{k} T_{z}} F^{*}\left(s_{\vartheta\left(a_{j}\right)}\right)\right)\right), \underset{\left(m\left(a_{1}\right), n\left(a_{1}\right)\right) \in h\left(a_{1}\right), \ldots,\left(m\left(a_{k}\right), n\left(a_{k}\right)\right) \in h\left(a_{k}\right)}{\cup}\right. \\
& \left.\left\{\frac{\sum_{j=1}^{k} F^{*}\left(s_{\vartheta\left(a_{j}\right)}\right) m_{j} T_{j}}{\sum_{j=1}^{k} F^{*}\left(s_{\vartheta\left(a_{j}\right)}\right) T_{j}}, \frac{\sum_{j=1}^{k} F^{*}\left(s_{\vartheta\left(a_{j}\right)}\right) n_{j} T_{j}}{\sum_{j=1}^{k} F^{*}\left(s_{\vartheta\left(a_{j}\right)}\right) T_{j}}\right\}\right\rangle
\end{aligned}
$$

Proof. Weon (28) 
(1) When $k=2$, since

$$
\begin{aligned}
& \frac{T_{1}}{\sum_{z=1}^{k} T_{z}} a_{1}=\left\langle F^{*-1}\left(\frac{T_{1}}{\sum_{z=1}^{k} T_{z}} F^{*}\left(s_{\vartheta\left(a_{1}\right)}\right)\right), h\left(a_{1}\right)\right\rangle \\
& \frac{T_{2}}{\sum_{z=1}^{k} T_{z}} a_{2}=\left\langle F^{*-1}\left(\frac{T_{2}}{\sum_{z=1}^{k} T_{z}} F^{*}\left(s_{\vartheta\left(a_{2}\right)}\right)\right), h\left(a_{2}\right)\right\rangle
\end{aligned}
$$

we have,

$$
\begin{aligned}
& \operatorname{HIFLPWA}\left(a_{1}, a_{2}\right) \frac{T_{1}}{\sum_{z=1}^{k} T_{z}} a_{1}+\frac{T_{2}}{\sum_{z=1}^{k} T_{z}} a_{2} \\
& =\left\langle F^{*-1}\left(\frac{T_{1}}{\sum_{z=1}^{k} T_{z}} F^{*}\left(s_{\vartheta\left(a_{1}\right)}\right)+\frac{T_{1}}{\sum_{z=1}^{k} T_{z}} F^{*}\left(s_{\vartheta\left(a_{2}\right)}\right)\right),{ }_{\left(m\left(a_{1}\right), n\left(a_{1}\right)\right) \in h\left(a_{1}\right),\left(m\left(a_{2}\right), n\left(a_{2}\right)\right) \in h\left(a_{2}\right)}^{\cup}\right. \\
& \left.\left\{\frac{F^{*}\left(s_{\vartheta\left(a_{1}\right)}\right) m_{1} T_{1}+F^{*}\left(s_{\vartheta\left(a_{2}\right)}\right) m_{2} T_{2}}{F^{*}\left(s_{\vartheta\left(a_{1}\right)}\right) T_{1}+F^{*}\left(s_{\vartheta\left(a_{2}\right)}\right) T_{2}}, \frac{F^{*}\left(s_{\vartheta\left(a_{1}\right)}\right) n_{1} T_{1}+F^{*}\left(s_{\vartheta\left(a_{2}\right)}\right) n_{2} T_{2}}{F^{*}\left(s_{\vartheta\left(a_{1}\right)}\right) T_{1}+F^{*}\left(s_{\vartheta}\left(a_{2}\right)\right) T_{2}}\right\}\right\rangle
\end{aligned}
$$

Thus, Equation (28) holds for $k=2$.

(2) Let us assume that Equation (28) is true for $k=g$, i.e.,

$$
\begin{aligned}
\operatorname{HIFLPWA}\left(a_{1}, a_{2}, \ldots, a_{g}\right) & =\frac{T_{1}}{\sum_{z=1}^{k} T_{z}} a_{1}+\frac{T_{2}}{\sum_{z=1}^{k} T_{z}} a_{2}+\ldots+\frac{T_{g}}{\sum_{z=1}^{k} T_{z}} a_{g} \\
& =\left\langle F^{*-1}\left(\sum_{j=1}^{g}\left(\frac{T_{j}}{\sum_{z=1}^{k} T_{z}} F^{*}\left(s_{\vartheta\left(a_{j}\right)}\right)\right)\right),{ }_{\left(m\left(a_{1}\right), n\left(a_{1}\right)\right) \in h\left(a_{1}\right), \ldots,\left(m\left(a_{g}\right), n\left(a_{g}\right)\right) \in h\left(a_{g}\right)}\right. \\
& \left.\left\{\frac{\sum_{j=1}^{g} F^{*}\left(s_{\vartheta\left(a_{j}\right.}\right) m_{j} T_{j}}{\sum_{j=1}^{g} F^{*}\left(s_{\vartheta\left(a_{j}\right)}\right) T_{j}}, \frac{\sum_{j=1}^{g} F^{*}\left(s_{\vartheta\left(a_{j}\right)}\right) n_{j} T_{j}}{\sum_{j=1}^{g} F^{*}\left(s_{\vartheta\left(a_{j}\right)}\right) T_{j}}\right\}\right\rangle
\end{aligned}
$$
we have

When $k=g+1$, then by the operations defined for HIFLNs and by Equations (29) and (30),

$$
\begin{aligned}
& \operatorname{HIFLPWA}\left(a_{1}, a_{2}, \ldots, a_{g+1}\right)=\left\langle F^{*-1}\left(\sum_{j=1}^{g}\left(\frac{T_{j}}{\sum_{z=1}^{k} T_{z}} F^{*}\left(s_{\vartheta \vartheta\left(a_{j}\right)}\right)\right)\right), \underset{\left(m\left(a_{1}\right), n\left(a_{1}\right)\right) \in h\left(a_{1}\right), \ldots,\left(m\left(a_{g}\right), n\left(a_{g}\right)\right) \in h\left(a_{g}\right)}{U}\right. \\
&\left\{\frac{\sum_{j=1}^{g} F^{*}\left(s_{\vartheta\left(a_{j}\right)}\right) m_{j} T_{j}}{\sum_{j=1}^{g} F^{*}\left(s_{\vartheta\left(a_{j}\right)}\right) T_{j}}, \frac{\sum_{j=1}^{g} F^{*}\left(s_{\vartheta\left(a_{j}\right)}\right) n_{j} T_{j}}{\sum_{j=1}^{g} F^{*}\left(s_{\vartheta\left(a_{j}\right)}\right) T_{j}}\right\}+\left\langle F^{*-1}\left(\frac{T_{g+1}}{\sum_{z=1}^{k} T_{z}} F^{*}\left(s_{\vartheta\left(a_{g+1}\right)}\right)\right), h\left(a_{g+1}\right)\right\rangle \\
&=\left\langle F^{*-1}\left(\sum_{j=1}^{g+1}\left(\frac{T_{j}}{\sum_{z=1}^{k} T_{z}} F^{*}\left(s_{\vartheta\left(a_{j}\right)}\right)\right)\right),\right. \cup\left(m\left(a_{1}\right), n\left(a_{1}\right)\right) \in h\left(a_{1}\right), \ldots,\left(m\left(a_{g+1}\right), n\left(a_{g+1}\right)\right) \in h\left(a_{g+1}\right) \\
&\left.\left\{\frac{\sum_{j=1}^{g+1} F^{*}\left(s_{\vartheta\left(a_{j}\right)}\right) m_{j} T_{j}}{\sum_{j=1}^{g+1} F^{*}\left(s_{\vartheta\left(a_{j}\right)}\right) T_{j}}, \frac{\sum_{j=1}^{g+1} F^{*}\left(s_{\vartheta}\left(a_{j}\right)\right) n_{j} T_{j}}{\sum_{j=1}^{g+1} F^{*}\left(s_{\vartheta\left(a_{j}\right)}\right) T_{j}}\right\}\right\rangle
\end{aligned}
$$

Therefore, Equation (28) is true for all $k$. Hence,

$$
\begin{aligned}
\operatorname{HIFLPWA}\left(a_{1}, a_{2}, \ldots, a_{k}\right) & =\frac{T_{1}}{\sum_{z=1}^{k} T_{z}} a_{1}+\frac{T_{2}}{\sum_{z=1}^{k} T_{z}} a_{2}+\ldots+\frac{T_{k}}{\sum_{z=1}^{k} T_{z}} a_{k} \\
& =\left\langle F^{*-1}\left(\sum_{j=1}^{k}\left(\frac{T_{j}}{\sum_{z=1}^{k} T_{z}} F^{*}\left(s_{\vartheta\left(a_{j}\right)}\right)\right)\right), \underset{\left(m\left(a_{1}\right), n\left(a_{1}\right)\right) \in h\left(a_{1}\right), \ldots,\left(m\left(a_{k}\right), n\left(a_{k}\right)\right) \in h\left(a_{k}\right)}{\cup}\right. \\
& \left.\left\{\frac{\sum_{j=1}^{k} F^{*}\left(s_{\vartheta\left(a_{j}\right)}\right) m_{j} T_{j}}{\sum_{j=1}^{k} F^{*}\left(s_{\vartheta\left(a_{j}\right)}\right) T_{j}}, \frac{\sum_{j=1}^{k} F^{*}\left(s_{\vartheta\left(a_{j}\right)}\right) n_{j} T_{j}}{\sum_{j=1}^{k} F^{*}\left(s_{\vartheta\left(a_{j}\right)}\right) T_{j}}\right\}\right\rangle
\end{aligned}
$$

Q.E.D.

Next, we give some properties of the HIFLPWA operator. 
Theorem 2 (boundedness). Let $a_{j}(j=1,2, \ldots, k)$ be a group of HIFLNs. If

$$
\begin{aligned}
& a^{-}=\left\langle\min _{j}\left\{s_{\vartheta\left(a_{j}\right)}\right\},\left(m^{-}, n^{+}\right)\right\rangle, a^{-}=\left\langle\max _{j}\left\{s_{\vartheta\left(a_{j}\right)}\right\},\left(m^{+}, n^{-}\right)\right\rangle, \\
& \text {where }\left(m^{-}, n^{+}\right)=\underset{\left(m_{j}, n_{j}\right) \in h\left(a_{1}\right), h\left(a_{2}\right), \ldots, h\left(a_{k}\right)}{\cup}\left\{\left(\min m_{j}, \max n_{j}\right)\right\},\left(m^{+}, n^{-}\right)=\underset{\left(m_{j}, n_{j}\right) \in h\left(a_{1}\right), h\left(a_{2}\right), \ldots, h\left(a_{k}\right)}{\cup}\left\{\left(\max m_{j}, \min n_{j}\right)\right\}, \text { then } \\
& S\left(a^{-}\right) \leq S\left(\operatorname{HIFLPWA}\left(a_{1}, a_{2}, \ldots, a_{k}\right) \leq S\left(a^{+}\right) .\right.
\end{aligned}
$$

Proof. Let HIFLPWA $\left(a_{1}, a_{2}, \ldots, a_{k}\right)=a=\left\langle s_{\vartheta(a)}, h(a)\right\rangle$, and then $S(a)=F^{*}\left(s_{\vartheta(a)}\right) \times C(h(a))$.

For LT $\min _{j}\left\{s_{\vartheta\left(a_{j}\right)}\right\} \leq s_{\vartheta\left(a_{j}\right)} \leq \max _{j}\left\{s_{\vartheta\left(a_{j}\right)}\right\}$ for all $j$, we have

$$
\begin{aligned}
\min _{j}\left\{s_{\vartheta\left(a_{j}\right)}\right\} & =F^{*-1}\left(\sum_{l=1}^{k}\left(\frac{T_{l}}{\sum_{z=1}^{k} T_{z}} F^{*}\left(\min _{j}\left\{s_{\vartheta\left(a_{j}\right)}\right\}\right)\right)\right) \\
& \leq F^{*-1}\left(\sum_{l=1}^{k}\left(\frac{T_{l}}{\sum_{z=1}^{k} T_{z}} F^{*}\left(s_{\vartheta}\left(a_{l}\right)\right)\right)\right)=s_{\vartheta(a)} \\
& \leq F^{*-1}\left(\sum_{l=1}^{k}\left(\frac{T_{l}}{\sum_{z=1}^{k} T_{z}} F^{*}\left(\max _{j}\left\{s_{\vartheta\left(a_{j}\right)}\right\}\right)\right)\right) \\
& =\max _{j}\left\{s_{\vartheta\left(a_{j}\right)}\right\}
\end{aligned}
$$

and then $\min _{j}\left\{s_{\vartheta\left(a_{j}\right)}\right\} \leq s_{\vartheta\left(a_{j}\right)} \leq \max _{j}\left\{s_{\vartheta\left(a_{j}\right)}\right\}$.

Similarly, since

$$
\begin{aligned}
& C\left(m^{-}, n^{+}\right)=\left(m^{-}, n^{+}\right) \\
& =C\left(\frac{\sum_{j=1}^{k} F^{*}\left(s_{\vartheta\left(a_{j}\right)}\right) T_{j} \cdot m^{-}}{\sum_{j=1}^{k} F^{*}\left(s_{\vartheta\left(a_{j}\right)}\right) T_{j}}, \frac{\sum_{j=1}^{k} F^{*}\left(s_{\vartheta\left(a_{j}\right)}\right) T_{j} \cdot n^{+}}{\sum_{j=1}^{k} F^{*}\left(s_{\vartheta\left(a_{j}\right.}\right) T_{j}}\right) \\
& \leq C\left(m_{\left(m\left(a_{1}\right), n\left(a_{1}\right)\right) \in h\left(a_{1}\right), \ldots,\left(m\left(a_{k}\right), n\left(a_{k}\right)\right) \in h\left(a_{k}\right)} \frac{\sum_{j=1}^{k} F^{*}\left(s_{\vartheta\left(a_{j}\right)}\right) T_{j} \cdot m_{j}}{\sum_{j=1}^{k} F^{*}\left(s_{\vartheta\left(a_{j}\right)}\right) T_{j}}, \frac{\sum_{j=1}^{k} F^{*}\left(s_{\vartheta\left(a_{j}\right)}\right) T_{j} \cdot n_{j}}{\sum_{j=1}^{k} F^{*}\left(s_{\vartheta}\left(a_{j}\right)\right) T_{j}}\right) \\
& =C(h(a)) \\
& \leq C\left(\frac{\sum_{j=1}^{k} F^{*}\left(s_{\vartheta\left(a_{j}\right)}\right) T_{j} \cdot m^{+}}{\sum_{j=1}^{k} F^{*}\left(s_{\vartheta\left(a_{j}\right)}\right) T_{j}}, \frac{\sum_{j=1}^{k} F^{*}\left(s_{\vartheta\left(a_{j}\right)}\right) T_{j} \cdot n^{-}}{\sum_{j=1}^{k} F^{*}\left(s_{\vartheta\left(a_{j}\right)}\right) T_{j}}\right) \\
& =\left(m^{+}, n^{-}\right)=C\left(m^{+}, n^{-}\right)
\end{aligned}
$$

Therefore,

$$
\begin{aligned}
S\left(a^{-}\right)=F^{*} & \left(\min _{j}\left\{s_{\vartheta\left(a_{j}\right)}\right\}\right) \cdot C\left(\left(m^{-}, n^{+}\right)\right) \leq F^{*}\left(s_{\vartheta\left(a_{j}\right)}\right) \cdot C(h(a)) \\
& \leq F^{*}\left(\max _{j}\left\{s_{\vartheta\left(a_{j}\right)}\right\}\right) \cdot C\left(\left(m^{+}, n^{-}\right)\right)=S\left(a^{+}\right)
\end{aligned}
$$

Thus, $S\left(a^{-}\right) \leq S\left(\operatorname{HIFLPWA}\left(a_{1}, a_{2}, \ldots, a_{k}\right) \leq S\left(a^{+}\right)\right.$.

Theorem 3 (commutativity). Suppose $a_{j}(j=1,2, \ldots, k)$ is a group of HIFLNs and $\left(\widehat{a}_{1}, \widehat{a}_{2}, \ldots, \widehat{a}_{k}\right)$ be any permutation of $\left(a_{1}, a_{2}, \ldots, a_{k}\right)$. Then,

$$
\operatorname{HIFLPWA}\left(a_{1}, a_{2}, \ldots, a_{k}\right)=\operatorname{HIFLPWA}\left(\widehat{a}_{1}, \widehat{a}_{2}, \ldots, \widehat{a}_{k}\right) .
$$


The weight of $a_{j}$ is determined by the priority and value of $a_{j}$, and will not be affected by its position in the permutation. Thus, Theorem 3 is obvious, and therefore the proof of Theorem 3 is omitted here.

It should be noted that the HIFLPWA operator is idempotent in a special case. Take $a=\left\langle s_{3},(0.4,0.5)\right\rangle$ $=a_{1}=a_{2}$, then $\operatorname{HIFLPWA}\left(a_{1}, a_{2}\right)=\left\langle s_{3},(0.4,0.5)\right\rangle$. That is to say, if all the HIFNs are equal and they consists of single IFN, then HIFLPWA operator is idempotent; otherwise, it cannot satisfy idempotency. For example, take $a=\left\langle s_{2},(0.3,0.2),(0.4,0.3)\right\rangle=a_{1}=a_{2}$, then $\operatorname{HIFLPWA}\left(a_{1}, a_{2}\right)=$ $\left\langle s_{1.98},(0.3871,0.2580),(0.4516,0.3622),(0.4516,0.3226),(0.5161,0.3871)\right\rangle \neq a$. In addition, the HIFLPWA operator does not satisfy monotonicity, because the weights will be recalculated and vary if the values used in the HIFLPWA operator are changed. It is difficult to consider monotonic property when the parameters are irregularly variable.

\subsection{The HIFLPWG Operator}

In this section, we define HIFLPWG operator for HIFLNs and discuss some related properties of this operator.

Definition 18. Let $a_{j}(j=1,2, \ldots, k)$ be a group of HIFLNs, then the HIFLPWG operator is given as follows:

$$
\operatorname{HIFLPWG}\left(a_{1}, a_{2}, \ldots, a_{n}\right)=a_{1}^{\frac{T_{1}}{\sum_{z=1}^{k} T_{z}}} \times a^{\frac{T_{2}}{\sum_{z=1}^{k} T_{z}}} \times \ldots \times a_{k}^{\frac{T_{k}}{\sum_{z=1}^{k} T_{z}}}=\bigotimes_{j=1}^{k} a_{j}^{\frac{T_{j}}{\sum_{z=1}^{k} T_{z}}}
$$

where $T_{j}=\prod_{l=1}^{j-1} S\left(a_{l}\right)(j=2,3, \ldots, k), T_{1}=1$, and $S\left(a_{l}\right)$ is the SF of $a_{l}$.

The HIFLPWG operator has the same properties as that of HIFLPWA operator.

Theorem 4. Suppose $a_{j}(j=1,2, \ldots, k)$ is a group of HIFLNs. Then, the aggregated result obtained by Definition 18 is also a HIFLN, and

$$
\begin{aligned}
\operatorname{HIFLPWG}\left(a_{1}, a_{2}, \ldots, a_{k}\right)= & \left\langle F^{*-1}\left(\prod_{j=1}^{k}\left(F^{*}\left(s_{\vartheta}\left(a_{j}\right)\right)\right)^{\frac{T_{j}}{\sum_{z=1}^{k} T_{z}}}\right), \underset{\left(m\left(a_{1}\right), n\left(a_{1}\right)\right) \in h\left(a_{1}\right), \ldots,\left(m\left(a_{k}\right), n\left(a_{k}\right)\right) \in h\left(a_{k}\right)}{U}\right. \\
& \left.\left\{\prod_{j=1}^{k}\left(m_{j}\right)^{\frac{T_{j}}{\sum_{z=1}^{k} T_{z}}}, 1-\prod_{j=1}^{k}\left(1-n_{j}\right)^{\frac{T_{j}}{\sum_{z=1}^{k} T_{z}}}\right\}\right\rangle
\end{aligned}
$$

where $T_{j}=\prod_{l=1}^{j-1} S\left(a_{l}\right)(j=2,3, \ldots, k), T_{1}=1$, and $S\left(a_{l}\right)$ is the SF of $a_{l}$.

The proof is the same as Theorem 1 , thus it is omitted here.

Theorem 5 (Boundedness). Suppose $a_{j}(j=1,2, \ldots, k)$ is a group of HIFLNs. If

$$
a^{-}=\left\langle\min _{j}\left\{s_{\vartheta\left(a_{j}\right)}\right\},\left(m^{-}, n^{+}\right)\right\rangle, a^{-}=\left\langle\max _{j}\left\{s_{\vartheta\left(a_{j}\right)}\right\},\left(m^{+}, n^{-}\right)\right\rangle
$$

where

$$
\left(m^{-}, n^{+}\right)=\underset{\left(m_{j}, n_{j}\right) \in h\left(a_{1}\right), h\left(a_{2}\right), \ldots, h\left(a_{k}\right)}{\cup}\left\{\left(\min m_{j}, \max n_{j}\right)\right\},\left(m^{+}, n^{-}\right)=\underset{\left(m_{j}, n_{j}\right) \in h\left(a_{1}\right), h\left(a_{2}\right), \ldots, h\left(a_{k}\right)}{\cup}\left\{\left(\max m_{j}, \min n_{j}\right)\right\},
$$

then,

$$
S\left(a^{-}\right) \leq S\left(\operatorname{HIFLPWG}\left(a_{1}, a_{2}, \ldots, a_{k}\right) \leq S\left(a^{+}\right) .\right.
$$


Proof. The proof is same as Theorem 2, thus it is omitted here.

Theorem 6 (commutativity). Suppose $a_{j}(j=1,2, \ldots, k)$ is a group of HIFLNs and $\left(\widehat{a}_{1}, \widehat{a}_{2}, \ldots, \widehat{a}_{k}\right)$ be any permutation of $\left(a_{1}, a_{2}, \ldots, a_{k}\right)$. Then

$$
\operatorname{HIFLPWG}\left(a_{1}, a_{2}, \ldots, a_{k}\right)=\operatorname{HIFLPWG}\left(\widehat{a}_{1}, \widehat{a}_{2}, \ldots, \widehat{a}_{k}\right)
$$

The weight of $a_{j}$ is only determined by the priority and value of $a_{j}$, and it will not be affected by the position order in the permutation. Thus, Theorem 6 is obvious, and therefore the proof is omitted here.

It should be noted that the HIFLPWG operator is idempotent in a special case. Take $a=\left\langle s_{3},(0.4,0.5)\right\rangle$ $=a_{1}=a_{2}$, then HIFLPWG $\left(a_{1}, a_{2}\right)=\left\langle s_{3},(0.4,0.5)\right\rangle$. That is to say, all the HIFLNs are equal and consist of single IFN, then HIFLPWG operator is idempotent, otherwise it cannot satisfy the property of idempotency. Similar to HIFPWA operator, the property of monotonicity cannot be considered for HIFPWG operator due to the same reason.

\section{MAMD Method with HIFLNs}

In this part, the HIFLPWA and HIFPWG operators will be used to solve the MADM problems with HIFLNs.

\subsection{Decision Steps Based on the HIFLPWA and HIFPWG Operators}

For MADM problems with HIFNs, let us suppose that there is a set of attributes denoted by $C=\left\{c_{1}, c_{2}, \ldots, c_{g}\right\}$ and the prioritization relationship between them is $c_{1}>c_{2}>\ldots>c_{g}$. Each attribute in $c_{z}$ has a higher priority than the attribute $c_{l}$ if $z<l$. In addition, there is a set of alternatives denoted by $\left\{m_{1}, m_{2}, \ldots, m_{k}\right\}$, and the DMs gave the evaluation value of the attribute $c_{j}$ for alternative $m_{i}$ by the HIFLN $c_{j}\left(m_{i}\right),(i=1,2, \ldots, k, j=1,2, \ldots, g)$. Suppose that $d=c_{j}\left(m_{i}\right)$ is the evaluation matrix. Finally, a ranking result for the alternatives is required.

The method for above MADM problem involves following main steps:

(1) Normalize the evaluation matrix.

Generally, there are two common types of attributes in MADM problems: maximizing attributes and minimizing attributes. To establish a uniform attribute types, the minimizing attributes are converted into maximizing attributes using the negation operation in Equation (22).

(2) Calculate $T_{i j}(i, 1,2, \ldots, k, j=1,2, \ldots, g)$.

(3) Get the overall assessment values $M_{i}(i=1,2, \ldots, k)$ of $m_{i}$ by applying HIFLPWA or HIFLPWG operator, i.e.,

$$
\begin{aligned}
M_{i} & =\operatorname{HIFLPWA}\left(c_{1}\left(m_{1}\right), c_{2}\left(m_{2}\right), \ldots, c_{g}\left(m_{i}\right)\right) \text { or } \\
& =\operatorname{HIFLPWG}\left(c_{1}\left(m_{1}\right), c_{2}\left(m_{2}\right), \ldots, c_{g}\left(m_{i}\right)\right)
\end{aligned}
$$

(4) Obtain the score and accuracy values of $M_{i}$.

(5) Rank all the alternatives based on score and accuracy values, and then select the best alternative.

\subsection{Illustrative Example}

Background: The following case is adopted from [36].

Example 4. Talented people are the most valued asset in the knowledge economy, so it is very important for a company to choose the right man to do the right thing to improve performance. Now, there is an opening for the supervisor position, whose responsibility is to do production planning and inventory control, and then to optimize supply chain system. By the initial examination, there are five candidates $M_{i}(i=1,2,3,4,5)$ who will be further evaluated by experts based on four attribute: their attitude $c_{1}$, ability $c_{2}$, leadership $c_{3}$ and skills $c_{4}$. Based on the 
experience and knowledge obtained by past recruiting, we know there exist prioritization relationships between these four criteria, denoted by $c_{1}>c_{2}>c_{3}>c_{4}$. The experts evaluate five candidates $m_{i}(i=1,2,3,4,5)$ for four attributes $c_{j}(j=1,2,3,4)$ under the LTS $S=\left\{s_{0}=\right.$ very poor, $s_{1}=$ slightly poor, $s_{2}=$ poor, $s_{3}=$ fair, $s_{4}=$ slightly good, $s_{5}=$ good, $s_{6}=$ very good $\}$, and construct the following decision matrix $d=c_{j}\left(m_{i}\right)$ shown in Table 1. The goal is to select the best candidate.

Table 1. The decision matrix with HIFLNs for Example 4.

\begin{tabular}{ccccc}
\hline Candidates & $c_{1}$ & $c_{2}$ & $c_{3}$ & $c_{4}$ \\
\hline$m_{1}$ & $\left\langle s_{5},\left\{\begin{array}{c}(0.5,0.1), \\
(0.8,0.1)\end{array}\right\}\right\rangle$ & $\left\langle s_{5},\{(0.5,0.2)\}\right\rangle$ & $\left\langle s_{5},\left\{\begin{array}{c}(0.6,0.2), \\
(0.9,0.0)\end{array}\right\}\right\rangle$ & $\left\langle s_{5},\{(0.8,0.0)\}\right\rangle$ \\
$(0.2,0.6)$, & $\left\langle s_{2},\left\{\begin{array}{c}0.6,0.2) \\
m_{2}\end{array}\right.\right.$ & $\left\langle s_{4},\left\{\begin{array}{c}(0.4,0.5), \\
(0.6,0.2)\end{array}\right\}\right\rangle$ & $\left\langle s_{3},\{(0.7,0.1)\}\right\rangle$ & $\left\langle s_{4},\left\{\begin{array}{c}(0.4,0.5), \\
(0.6,0.2)\end{array}\right\}\right\rangle$ \\
$m_{3}$ & $\left\langle s_{3},\left\{\begin{array}{c}0.6,0.5), \\
(0.6,0.1)\end{array}\right\}\right\rangle$ & $\left\langle s_{4},\{(0.4,0.5)\}\right\rangle$ & $\left\langle s_{6},\left\{\begin{array}{c}(0.8,0.1), \\
(0.9,0.1)\end{array}\right\}\right\rangle$ & $\left\langle s_{4},\{(0.4,0.5)\}\right\rangle$ \\
$m_{4}$ & $\left\langle s_{4},\{(0.4,0.5)\}\right\rangle$ & $\left\langle s_{5},\left\{\begin{array}{c}(0.2,0.6), \\
(0.5,0.4)\end{array}\right\}\right\rangle$ & $\left\langle s_{3},\left\{\begin{array}{c}(0.6,0.2), \\
(0.7,0.1) \\
(0.4,0.5), \\
(0.7,0.1)\end{array}\right\}\right\rangle$ & $\left\langle s_{3},\{(0.5,0.4)\}\right\rangle$ \\
$m_{5}$ & $\left\langle s_{2},\left\{\begin{array}{c}(0.3,0.5),(0.5,0.4), \\
(0.5,0.3)\end{array}\right\}\right\rangle$ & $\left\langle s_{3,},\{(0.6,0.4)\}\right\rangle$ & $\left\langle s_{2},\{(0.9,0.1)\}\right\rangle$ \\
\hline
\end{tabular}

The steps are shown as follows.

Step 1. Normalize the decision matrix.

Because all attributes are all benefit type, this step is omitted.

Step 2. Calculate $T_{i j}(i=1,2,3,4,5, j=1,2,3,4)$, and get

$$
T_{i j}=\left(\begin{array}{cccc}
1 & 0.542 & 0.687 & 0.750 \\
1 & 0.383 & 0.4 & 0.383 \\
1 & 0.300 & 0.875 & 0.300 \\
1 & 0.312 & 0.375 & 0.275 \\
1 & 0.3 & 0.313 & 0.300
\end{array}\right)
$$

Step 3. Calculate the overall assessment values $M_{i}(i=1,2, \ldots, k)$ of $m_{i}$ by the HIFLPWA operator, we can get

$$
\begin{gathered}
M_{1}=\left\langle s_{5.00},\{(0.599,0.116),(0.668,0.070),(0.699,0.116),(0.769,0.070)\}\right\rangle, \\
M_{2}=\left\langle\begin{array}{c}
s_{2.892},\{(0.394,0.455),(0.443,0.382),(0.443,0.382),(0.492,0.308) \\
(0.521,0.328),(0.570,0.254),(0.570,0.254),(0.619,0.181)\}
\end{array}\right\rangle, \\
M_{3}=\left\langle s_{4.303},\{(0.597,0.303),(0.646,0.292),(0.654,0.190),(0.703,0.179)\}\right\rangle, \\
M_{4}=\left\langle s_{3.828},\{(0.399,0.465),(0.414,0.450),(0.441,0.444),(0.456,0.429)\}\right\rangle, \\
M_{5}=\left\langle\begin{array}{c}
s_{2.320},\{(0.463,0.426),(0.526,0.341),(0.553,0.381),(0.617,0.296) \\
(0.553,0.336),(0.617,0.251)\}
\end{array}\right\rangle .
\end{gathered}
$$

or by the HIFLPWG operator, we can get

$$
\begin{aligned}
M_{1} & =\left\langle s_{5.00},\{(0.587,0.120),(0.645,0.073),(0.687,0.120),(0.755,0.073)\}\right\rangle, \\
M_{2} & =\left\langle\begin{array}{c}
s_{2.755},\{(0.322,0.497),(0.346,0.454),(0.346,0.454),(0.372,0.406) \\
(0.535,0.308),(0.575,0.248),(0.575,0.248),(0.617,0.182)\}
\end{array}\right\rangle, \\
M_{3} & =\left\langle s_{3.733},\{(0.511,0.385),(0.533,0.385),(0.602,0.220),(0.628,0.220)\}\right\rangle, \\
M_{4} & =\left\langle s_{3.778},\{(0.399,0.458),(0.411,0.446),(0.446,0.439),(0.459,0.426)\}\right\rangle,
\end{aligned}
$$




$$
M_{5}=\left\langle\begin{array}{c}
s_{2.277},\{(0.416,0.436),(0.456,0.379),(0.544,0.379),(0.456,0.317) \\
(0.544,0.327),(0.596,0.259)\}
\end{array}\right\rangle
$$

Step 4. The SFs for the candidate $m_{i}(i=1,2,3,4,5)$ can be obtained, as listed in Table 2.

Step 5. The ranking order of the candidates $m_{i}(1,2,3,4,5)$ is given in Table 2.

Table 2. SFs and ranking orders of the candidates.

\begin{tabular}{cc}
\hline HIFLPWA & HIFLPWG \\
\hline$S\left(M_{1}\right) 0.6627$ & 0.6550 \\
$S\left(M_{2}\right) 0.2864$ & 0.2551 \\
$S\left(M_{3}\right) 0.5053$ & 0.3939 \\
$S\left(M_{4}\right) 0.3128$ & 0.3106 \\
$S\left(M_{5}\right) 0.2352$ & 0.2187 \\
Ranking order $m_{1}>m_{3}>m_{4}>m_{2}>m_{5}$ & $m_{1}>m_{3}>m_{4}>m_{2}>m_{5}$ \\
\hline
\end{tabular}

In Table 2, one can see that the ranking orders obtained through HIFLPWA and HIFLPWG operators are the same, and $m_{1}$ is the best candidate, while $m_{5}$ is the worst candidate.

To show the influence on the ranking results from the different LSFs, we adopt different LSFs in Steps 2-4 to obtain the SFs and ranking orders, which are listed in Table 3.

Table 3. Ranging results from the different LSFs.

\begin{tabular}{cccc}
\hline LSFs & HIFLPWA & HIFLPWG & Ranking Order \\
\hline \multirow{2}{*}{$F_{2}^{*}\left(s_{j}\right)$} & $S_{1}=0.6161, S_{2}=0.2860$, & $S_{1}=0.6090, S_{2}=0.2632$, & $m_{1}>m_{3}>m_{4}>$ \\
& $S_{3}=0.5043, S_{4}=0.3009$, & $S_{3}=0.3908, S_{4}=0.2964$, & $m_{2}>m_{5}$ \\
$S_{5}=0.2588$ & $S_{5}=0.2434$ & \\
$F_{3}^{*}\left(s_{j}\right)$ & $S_{1}=0.6761, S_{2}=0.2778$, & $S_{1}=0.6682, S_{2}=0.2442$, & $m_{1}>m_{3}>m_{4}>$ \\
& $S_{3}=0.5059, S_{4}=0.3182$, & $S_{3}=0.3953, S_{4}=0.3172$, & $m_{2}>m_{5}$ \\
& $S_{5}=0.2250$ & $S_{5}=0.2075$ &
\end{tabular}

In Table 3, we can know that the same ranking result $m_{1}>m_{3}>m_{4}>m_{2}>m_{5}$ was obtained when different LSFs were used.

\subsection{Validity Verification of the Proposed Methods}

In this part, we verify the validity of the proposed methods; let us consider another example adapted from [37], with some different data.

Example 5. An investment enterprise wants to invest in the best company, and it must invest its money in one of the following four possible companies: $m_{1}$ is a car company; $m_{2}$ is food company; $m_{3}$ is a computer company; and $m_{4}$ is an arms company. Based on the LTS $S=\left\{s_{0}, s_{1}, s_{2}, s_{3}, s_{4}, s_{5}, s_{7}\right\}$, the decision makers should consider the following four attributes: $c_{1}$ is the risk factor; $c_{2}$ is the growth factor; $c_{3}$ is the social political factor; and $c_{4}$ is the environmental factor. Based on history analysis, prioritization relationships exist between these four attributes, denoted by $c_{1}>c_{2}>c_{3}>c_{4}$. The assessment values of the companies are represented by HIFLNs, and the decision matrix is constructed, as shown in Table 4. Then, the proposed approach is utilized to rank the companies. 
Table 4. The decision matrixof Example 5.

\begin{tabular}{cccc}
\hline$c_{1}$ & $c_{2}$ & $c_{3}$ & $c_{4}$ \\
\hline$m_{1}\left\langle s_{2},\left\{\begin{array}{c}(0.4,0.4), \\
(0.3,0.5)\end{array}\right\}\right\rangle$ & $\left\langle s_{4},\left\{\begin{array}{c}(0.6,0.1), \\
(0.4,0.3)\end{array}\right\}\right\rangle$ & $\left\langle s_{3},\left\{\begin{array}{c}(0.4,0.5), \\
(0.7,0.2)\end{array}\right\}\right\rangle$ & $\left\langle s_{4},\{(0.5,0.3)\}\right\rangle$ \\
$m_{2}\left\langle s_{3},\{(0.4,0.3)\}\right\rangle$ & $\left\langle s_{5},\left\{\begin{array}{c}(0.7,0.1), \\
(0.6,0.2)\end{array}\right\}\right\rangle$ & $\left\langle s_{4},\{(0.8,0.1)\}\right\rangle$ & $\left\langle s_{5},\left\{\begin{array}{c}(0.6,0.1), \\
(0.7,0.2)\end{array}\right\}\right\rangle$ \\
$m_{3}\left\langle s_{2},\left\{\begin{array}{c}(0.6,0.2), \\
(0.4,0.3)\end{array}\right\}\right\rangle$ & $\left\langle s_{4},\{(0.7,0.3)\}\right\rangle$ & $\left\langle s_{3},\left\{\begin{array}{c}(0.6,0.2), \\
(0.5,0.3)\end{array}\right\}\right\rangle$ & $\left\langle s_{5},\{(0.4,0.2)\}\right\rangle$ \\
$m_{4}\left\langle s_{3},\{(0.2,0.4)\}\right\rangle$ & $\left\langle s_{3},\left\{\begin{array}{c}(0.6,0.1), \\
(0.5,0.2)\end{array}\right\}\right\rangle$ & $\left\langle s_{4},\{(0.5,0.4)\}\right\rangle$ & $\left\langle s_{4},\left\{\begin{array}{c}(0.6,0.3), \\
(0.5,0.4)\end{array}\right\}\right\rangle$ \\
\hline
\end{tabular}

Then, the decision steps are shown as follows.

Step 1. Because all attributes are all benefit type, initialization is omitted.

Step 2. Calculate $T_{i j}(i, j=1,2,3,4)$, we have

$$
T_{i j}=\left(\begin{array}{cccc}
1 & 0.4333 & 0.3 & 0.4 \\
1 & 0.625 & 0.567 & 0.625 \\
1 & 0.467 & 0.325 & 0.4 \\
1 & 0.7 & 0.367 & 0.4
\end{array}\right)
$$

Step 3. Calculate the overall assessment values $M_{i}(i=1,2, \ldots, k)$ of $m_{i}$ by the HIFLPWA operator, we can get

$$
\begin{gathered}
M_{1}=\left\langle s_{2.922},\left\{\begin{array}{c}
(0.481,0.305),(0.525,0.262),(0.426,0.361),(0.469,0.318), \\
(0.449,0.337),(0.493,0.294),(0.394,0.393),(0.437,0.350)
\end{array}\right\}\right\rangle, \\
M_{2}=\left\langle s_{4.089},\{(0.614,0.152),(0.642,0.179),(0.587,0.317),(0.614,0.232)\}\right\rangle, \\
M_{3}=\left\langle s_{2.939},\{(0.579,0.229),(0.564,0.244),(0.517,0.260),(0.502,0.275)\}\right\rangle, \\
M_{4}=\left\langle s_{3.311},\{(0.435,0.303),(0.416,0.323),(0.409,0.329),(0.390,0.349)\}\right\rangle .
\end{gathered}
$$

or by the HIFLPWG operator, we can get

$$
\begin{gathered}
M_{1}=\left\langle s_{2.776},\left\{\begin{array}{c}
(0.453,0.346),(0.490,0.302),(0.417,0.379),(0.451,0.336) \\
(0.396,0.400),(0.428,0.359),(0.364,0.430),(0.394,0.391)
\end{array}\right\}\right\rangle, \\
M_{2}=\left\langle s_{3.988},\{(0.5697,0.1477),(0.5505,0.1722),(0.5697,0.2009),(0.5894,0.1723)\}\right\rangle, \\
M_{3}=\left\langle s_{2.794},\{(0.5758,0.2180),(0.5604,0.2315),(0.4785,0.2623),(0.4658,0.2786)\}\right\rangle, \\
M_{4}=\left\langle s_{3.281},\{(0.3741,0.2576),(0.3631,0.2699),(0.3552,0.3135),(0.3449,0.3407)\}\right\rangle .
\end{gathered}
$$

Step 4. Calculate the SFs for the company $m_{i}(i=1,2,3,4)$ as follows.

1. Use HIFLPWA operator, we have

$$
S\left(m_{1}\right)=0.275, S\left(m_{2}\right)=0.475, S\left(m_{3}\right)=0.316, S\left(m_{4}\right)=0.300 .
$$

2. Use the HIFLPWG operator, we have

$$
S\left(m_{1}\right)=0.244, S\left(m_{2}\right)=0.464, S\left(m_{3}\right)=0.296, S\left(m_{4}\right)=0.291 .
$$


Step 5. The ranking order of the companies $m_{i}(1,2,3,4,5)$ is shown as follows.

$$
m_{2}>m_{3}>m_{4}>m_{1}
$$

Thus, we can know that the ranking order obtained by the HIFLPWA operators is that $m_{2}$ is the best company and $m_{1}$ is the worst company among the four companies. Moreover, the ranking order obtained by the HIFLPWG operators is the same as that obtained from HIFLPWA operators. That is to say, $m_{2}$ is the best company while $m_{1}$ is the worst company.

Now, using the aggregation operators defined by Liu et al. [37], we get the following SFs. The SFs of the companies calculated by the HIFLWA operators are

$$
E\left(m_{1}\right)=0.0097, E\left(m_{2}\right)=0.0 .48, E\left(m_{3}\right)=0.025, E\left(m_{4}\right)=0.012 .
$$

Because $E\left(m_{2}\right)>E\left(m_{3}\right)>E\left(m_{4}\right)>E\left(m_{1}\right)$, thus, $m_{2}>m_{3}>m_{4}>m_{1}$.

The SFs calculated using HIFLWG operator are

$$
E\left(m_{1}\right)=0.0261, E\left(m_{2}\right)=0.263, E\left(m_{3}\right)=0.126, E\left(m_{4}\right)=0.0349 .
$$

Because $E\left(m_{2}\right)>E\left(m_{3}\right)>E\left(m_{4}\right)>E\left(m_{1}\right)$. Therefore, $m_{2}>m_{3}>m_{4}>m_{1}$.

Obviously, there are the same ranking results by the proposed methods in this article and the methods proposed by Liu et al. [37]. Hence, this shows validity of the proposed methods in this article.

\subsection{Comparative Analysis}

Example 6. To further illustrate the advantages of the proposed aggregation operator and SF defined in this article, we can solve the same Example 5 given above and the HIFLNs in this matrix are provided in Table 5.

Table 5. The new decision matrix of Example 6.

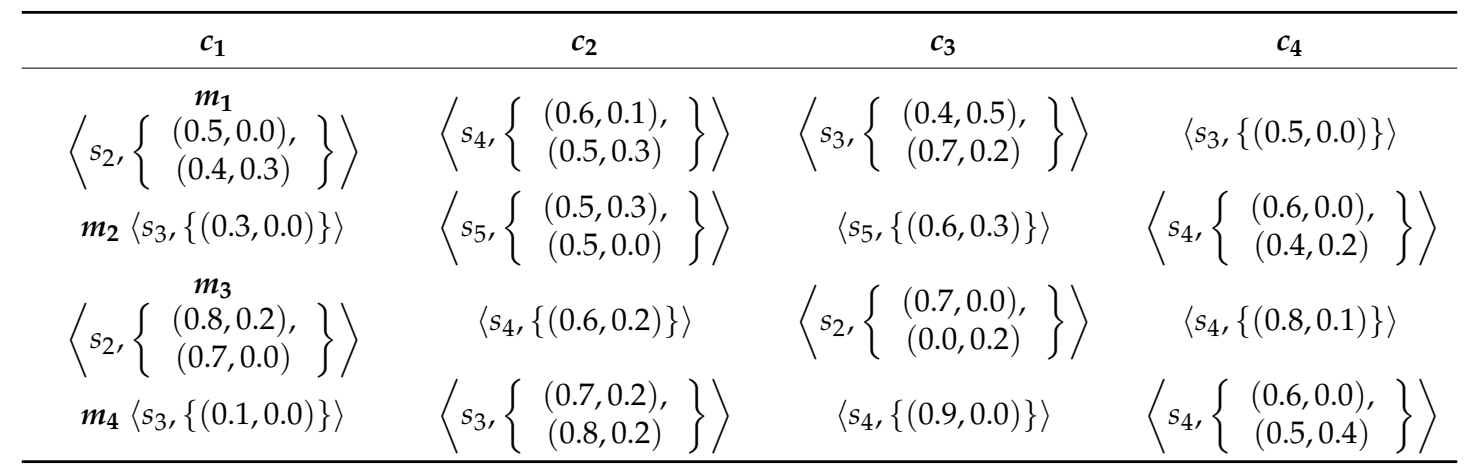

Now we use the aggregation operator defined on new operational laws in this article, and we get the following SFs and ranking orders given in Table 6.

Table 6. SFs and ranking orders of alternatives using HIFLPWA and HIFLPWG operators for Example 6.

\begin{tabular}{cccc}
\hline Alternatives & HIFLPWA & HIFLPWG & Ranking Order \\
\hline$m_{1}$ & $S\left(m_{1}\right)=0.306$ & $S\left(m_{1}\right)=0.288$ & \\
$m_{2}$ & $S\left(m_{2}\right)=0.434$ & $S\left(m_{2}\right)=0.467$ & $m_{2}>m_{4}>m_{3}>m_{1}$ \\
$m_{3}$ & $S\left(m_{3}\right)=0.382$ & $S\left(m_{3}\right)=0.303$ & \\
$m_{4}$ & $S\left(m_{4}\right)=0.412$ & $S\left(m_{4}\right)=0.381$ & \\
\hline
\end{tabular}

Then, we use the methods proposed by Liu et al. [37] to solve this example. The weights of the attributes are calculated using PA operator. 
By HIFLWA operator [37], the SFs of the alternative are calculated as follow:

$$
E\left(m_{1}\right)=0.0381, E\left(m_{2}\right)=0.0511, E\left(m_{3}\right)=0.0553, E\left(m_{4}\right)=0.0611
$$

$E\left(m_{4}\right)>E\left(m_{3}\right)>E\left(m_{2}\right)>E\left(m_{1}\right)$. Hence, $m_{4}>m_{3}>m_{2}>m_{1}$.

We can see that the ranking orders obtained by our methods and by the methods of Liu et al. [37] are totally different. We can analyze the reason as follows. As stated in Section 3.1, when we take the non-membership function equal to zero of the corresponding LTs, there is no effect of other grades on the overall aggregated values. That is to say, non-membership function of the LT does not play any role in the aggregation process. In this example, there are some zeroes in non-memberships of LTs, so the methods of Liu et al. [37] cannot give the reasonable ranking results, i.e., the ranking result $m_{4}>m_{3}>m_{2}>m_{1}$ is unreasonable and unacceptable, while the proposed methods in this article can solve this problem, and can produce a reasonable ranking result, i.e., the ranking result $m_{2}>m_{4}>m_{3}>m_{1}$ is reasonable.

Example 7. To further show the advantages of the SF defined in this article, we consider the same Example 5, and the HIFLNs given by the decision makers are listed in Table 7.

Table 7. The decision matrix of Example 7.

\begin{tabular}{cccc}
\hline$c_{1}$ & $c_{2}$ & $c_{3}$ & $c_{4}$ \\
\hline$\left\langle s_{2},\left\{\begin{array}{c}m_{1} \\
(0.5,0.0), \\
(0.4,0.3)\end{array}\right\}\right\rangle$ & $\left\langle s_{4},\left\{\begin{array}{c}(0.6,0.1), \\
(0.5,0.3)\end{array}\right\}\right\rangle$ & $\left\langle s_{3},\left\{\begin{array}{c}(0.4,0.5), \\
(0.7,0.2)\end{array}\right\}\right\rangle$ & $\left\langle s_{3},\{(0.5,0.0)\}\right\rangle$ \\
$m_{2}\left\langle s_{3},\{(0.3,0.0)\}\right\rangle$ & $\left\langle s_{5},\left\{\begin{array}{c}(0.5,0.3), \\
(0.5,0.0)\end{array}\right\}\right\rangle$ & $\left\langle s_{4},\{(0.6,0.3)\}\right\rangle$ & $\left\langle s_{5},\left\{\begin{array}{c}(0.6,0.0), \\
(0.4,0.2)\end{array}\right\}\right\rangle$ \\
$\left\langle s_{2},\left\{\begin{array}{c}m_{3}(0.8,0.2), \\
(0.7,0.0)\end{array}\right\}\right\rangle$ & $\left\langle s_{4},\{(0.6,0.2)\}\right\rangle$ & $\left\langle s_{3},\left\{\begin{array}{c}(0.7,0.0), \\
(0.0,0.2)\end{array}\right\}\right\rangle$ & $\left\langle s_{3},\{(0.8,0.1)\}\right\rangle$ \\
$m_{4}\left\langle s_{3},\{(0.1,0.0)\}\right\rangle$ & $\left\langle s_{3},\left\{\begin{array}{c}(0.7,0.2), \\
(0.8,0.2)\end{array}\right\}\right\rangle$ & $\left\langle s_{4},\{(0.9,0.0)\}\right\rangle$ & $\left\langle s_{4},\left\{\begin{array}{c}(0.6,0.0), \\
(0.5,0.4)\end{array}\right\}\right\rangle$ \\
\hline
\end{tabular}

Now, we use the aggregation operator defined on new operational laws in this article, and we get the following SFs and ranking orders listed in Table 8.

Table 8. SFs and ranking orders of alternatives using HIFLPWA and HIFLPWG operators for Example 7.

\begin{tabular}{cccc}
\hline Alternatives & HIFLPWA & HIFLPWG & Ranking Order \\
\hline$m_{1}$ & $S\left(m_{1}\right)=0.306$ & $S\left(m_{1}\right)=0.288$ & \\
$m_{2}$ & $S\left(m_{2}\right)=0.442$ & $S\left(m_{2}\right)=0.468$ & $m_{2}>m_{4}>m_{3}>m_{1}$ \\
$m_{3}$ & $S\left(m_{3}\right)=0.350$ & $S\left(m_{3}\right)=0.291$ & \\
$m_{4}$ & $S\left(m_{4}\right)=0.412$ & $S\left(m_{4}\right)=0.381$ & \\
\hline
\end{tabular}

Then, we use the methods proposed by Liu et al. [37] to solve this example.

By HIFLWA operator [37], the SFs of the alternative are calculated as follow:

$$
E\left(m_{1}\right)=0.0381, E\left(m_{2}\right)=0.0508, E\left(m_{3}\right)=0.0508, E\left(m_{4}\right)=0.0611
$$

$E\left(m_{4}\right)>E\left(m_{3}\right)=E\left(m_{2}\right)>E\left(m_{1}\right)$. Hence, $m_{4}>m_{3}=m_{2}>m_{1}$.

As we can see that the SFs of the alternatives $m_{2}$ and $m_{3}$ produced by the methods of Liu et al. [37] are equal, and we can distinguish which one is better than the other. While using the SF defined in this article shows that $m_{2}$ is better than $m_{3}$. This shows that the proposed SF in this article is better than that of proposed by Liu et al. [37]. 
Based on above comparison analysis, the proposed methods for MADM for HIFLNs have the following advantages.

Firstly, the HIFLNs can depict the assessment information of MADM problems more flexibly because they can generalize most expressions of existing fuzzy information. This is a prerequisite for guarantying accuracy of the final ranking.

Secondly, the operational laws defined for HIFLNs in this article are based on the LSFs. Different results should be obtained using different LSFs, and three special examples of LSFs are proposed by actual applications. Thus, DMs may choose or redefine the LSFs according to their preferences or actual situations.

Thirdly, the proposed HIFLPWA and HIFLPWG operators can handle MADM problems with different priority level criteria.

\section{Conclusions}

Considering the shortcomings in the existing studies, we proposed some new operational laws of HIFLNs based on the LSFs which provided a flexible way to express the qualitative evaluation information given by the DMs, and then we presented a new score function, accuracy function and comparative method for the HIFLNs. Moreover, two PA operators for HIFLNs, i.e., the HIFLPWA and HIFLPWG operators, were developed, which could consider the prioritized relationship among the aggregated arguments. Based on the proposed prioritized aggregation operators, we developed two MADM methods to process the decision making problems in which the attributes have the prioritized relationship and the attribute values take the form of HIFLNs. Finally, some numerical examples are demonstrated to show the effectiveness and practicality of the proposed methods. By comparison analysis with the existing methods for HIFLNs, the results indicated that the proposed methods in this article are more effective and general in solving MADM problem under the environment of the HIFLNs by considering the prioritized relationship among the attributes and adopting the LSFs.

In the future, we shall study some new aggregation operators for HIFSs, such as Bonferroni mean $[40,41]$ and Heronian mean $[38,39,45]$, or apply the proposed methods to solve real applications, such as the evaluations for population, resources and environment [46-49].

Acknowledgments: This paper is supported by the National Natural Science Foundation of China (Nos. 71771140 and 71471172), the Special Funds of Taishan Scholars Project of Shandong Province (No. ts201511045), Shandong Provincial Social Science Planning Project (Nos. 16CGLJ31 and 16CKJJ27), the Natural Science Foundation of Shandong Province (No. ZR2017MG007), the Teaching Reform Research Project of Undergraduate Colleges and Universities in Shandong Province (No. 2015Z057), and Key research and development program of Shandong Province (No. 2016GNC110016).

Author Contributions: All authors have contributed equally to this paper.

Conflicts of Interest: The authors declare no conflict of interest.

\section{References}

1. Mardani, A.; Jusoh, K.M.D.N.; Khalifah, Z.; Zakwan, N.; Valipour, A. Multiple criteria decision-making techniques and their applications-a review of the literature from 2000 to 2014. Econ. Res. Ekono. Istraž. 2015, 28, 516-571. [CrossRef]

2. $\mathrm{Xu}, \mathrm{Z}$. A method based on linguistic aggregation operators for group decision making with linguistic preference relations. Inf. Sci. 2004, 166, 19-30. [CrossRef]

3. $\mathrm{Xu}, \mathrm{Z}$. A note on linguistic hybrid arithmetic averaging operator in multiple attribute group decision making with linguistic information. Group Decis. Negot. 2006, 15, 593-604. [CrossRef]

4. Zadeh, L.A. Fuzzy sets. Inf. Control 1965, 8, 338-353. [CrossRef]

5. Atanassov, K.T. Intuitionistic fuzzy sets. Fuzzy Sets Syst. 1986, 20, 87-96. [CrossRef]

6. Miyamoto, S. Multisets and fuzzy multisets. In Soft Computing and Human-Centered Machine; Springer: Berlin/Heidelberg, Germany, 2000; pp. 9-33.

7. Mizumoto, M.; Tanaka, K. Some properties of fuzzy sets of type 2. Inf. Control 1976, 31, 312-340. [CrossRef] 
8. Torra, V. Hesitant fuzzy sets. Int. J. Intell. Syst. 2010, 25, 529-539. [CrossRef]

9. Chen, J.; Huang, X.; Tang, J. Distance measures for higher order dual hesitant fuzzy sets. Comput. Appl. Math. 2017, 1-23. [CrossRef]

10. Kumar, K.; Garg, H. TOPSIS method based on the connection number of set pair analysis under interval-valued intuitionistic fuzzy set environment. Comput. Appl. Math. 2017, 1-11. [CrossRef]

11. Wei, G.; Zhang, N. A multiple criteria hesitant fuzzy decision making with Shapley value-based VIKOR method. J. Intell. Fuzzy Syst. 2014, 26, 1065-1075.

12. Xu, G.L.; Wan, S.P.; Dong, J.Y. A hesitant fuzzy programming method for hybrid MADM with incomplete attribute weight information. Informatica 2016, 27, 863-892. [CrossRef]

13. Yu, D.; Zhang, W.; Huang, G. Dual hesitant fuzzy aggregation operators. Technol. Econ. Dev. Econ. 2016, 22, 194-209. [CrossRef]

14. Zhu, B.; Xu, Z.; Xia, M. Extended hesitant fuzzy sets. Technol. Econ. Deve. Econ. 2016, 22, 100-121. [CrossRef]

15. Chen, N.; Xu, Z.; Xia, M. Correlation coefficients of hesitant fuzzy sets and their applications to clustering analysis. Appl. Math. Model. 2013, 37, 2197-2211. [CrossRef]

16. Peng, D.H.; Gao, C.Y.; Gao, Z.F. Generalized hesitant fuzzy synergetic weighted distance measures and their application to multiple criteria decision-making. Appl. Math. Model. 2013, 37, 5837-5850. [CrossRef]

17. Xu, Z.; Xia, M. Distance and similarity measures for hesitant fuzzy sets. Inf. Sci. 2011, 181, $2128-2138$. [CrossRef]

18. Xu, Z.; Xia, M. Hesitant fuzzy entropy and cross-entropy and their use in multiattribute decision-making. Int. J. Intell. Syst. 2012, 27, 799-822. [CrossRef]

19. Liao, H.; Xu, Z. Subtraction and division operations over hesitant fuzzy sets. J. Intell. Fuzzy Syst. 2014, 27, 65-72.

20. Liao, H.; Xu, Z. Some new hybrid weighted aggregation operators under hesitant fuzzy multi-criteria decision making environment. J. Intell. Fuzzy Syst. 2014, 26, 1601-1617.

21. Yager, R.R. Prioritized aggregation operators. Int. J. Approx. Reason. 2008, 48, 263-274. [CrossRef]

22. Zhang, X. Hesitant fuzzy power aggregation operators and their application to multiple attribute group decision making. Inf. Sci. 2013, 234, 150-181. [CrossRef]

23. Zhu, B.; Xu, Z.; Xia, M. Dual hesitant fuzzy sets. J. Appl. Math. 2012, 2012, 1-13. [CrossRef]

24. Singh, P. Distance and similarity measures for multiple-attribute decision making with dual hesitant fuzzy sets. Comput. Appl. Math. 2017, 36, 111-126. [CrossRef]

25. Martínez, L.; Ruan, D.; Herrera, F.; Herrera-Viedma, E.; Wang, P.P. Linguistic decision making: Tools and applications. Inf. Sci. 2009, 179, 2297-2298. [CrossRef]

26. Herrera, F.; Herrera-Viedma, E. Linguistic decision analysis: Steps for solving decision problems under linguistic information. Fuzzy Sets syst. 2000, 115, 67-82. [CrossRef]

27. Liu, P.; He, L.; Yu, X.C. Generalized hybrid aggregation operators based on the 2-dimension uncertain linguistic information for multiple attribute group decision making. Group Decis. Negot. 2016, 25, 103-126. [CrossRef]

28. Liu, P.; Teng, F. An extended TODIM method for multiple attribute group decision-making based on 2-dimension uncertain linguistic variable. Complexity 2016, 21, 20-30. [CrossRef]

29. Rodríguez, R.M.; Martínez, L.; Herrera, F. A group decision making model dealing with comparative linguistic expressions based on hesitant fuzzy linguistic term sets. Inf. Sci. 2013, 241, 28-42. [CrossRef]

30. Zadeh, L.A. The concept of a linguistic variable and its application to approximate reasoning-I. Inf. Sci. 1975, 8, 199-249. [CrossRef]

31. Rodríguez, R.M.; Martínez, L.; Herrera, F. Hesitant fuzzy linguistic term sets. In Foundations of Intelligent Systems; Springer: Heidelberg/Berlin, Germany, 2011; pp. 287-295.

32. Rodríguez, R.M.; Martínez, L.; Herrera, F. Hesitant fuzzy linguistic term sets for decision making. IEEE Trans. Fuzzy Syst. 2012, 20, 109-119. [CrossRef]

33. Lin, R.; Zhao, X.; Wei, G. Models for selecting an ERP system with hesitant fuzzy linguistic information. J. Intell. Fuzzy Syst. 2014, 26, 2155-2165.

34. Wu, J.T.; Wang, J.T.; Wang, J.Q.J.; Zhang, H.Y.; Chen, X.H. Hesitant Fuzzy Linguistic Multicriteria Decision-Making Method Based on Generalized Prioritized Aggregation Operator. Sci. World J. 2014, 2014, 1-20. [CrossRef] [PubMed] 
35. Liu, P. Special issue "Intuitionistic fuzzy theory and its application in economy, technology and management". Technol. Econ. Dev. Econ. 2016, 22, 327-335. [CrossRef]

36. Yu, D.J. Multi-criteria decision making based on generalized prioritized aggregation operators under intuitionistic fuzzy environment. Int. J. Fuzzy Syst. 2013, 15, 47-54.

37. Liu, X.; Ju, Y.; Yang, S. Hesitant intuitionistic fuzzy linguistic aggregation operators and their applications to multiple attribute decision making. J. Intell. Fuzzy Syst. 2014, 27, 1187-1201.

38. Liu, P. Multiple attribute group decision making method based on interval-valued intuitionistic fuzzy power Heronian aggregation operators. Comput. Ind. Eng. 2007, 108, 199-212. [CrossRef]

39. Liu, P.; Chen, S.M. Group decision making based on Heronian aggregation operators of intuitionistic fuzzy numbers. IEEE Trans. Cybern. 2017, 47, 2514-2530. [CrossRef] [PubMed]

40. Liu, P.; Chen, S.M.; Liu, J. Some intuitionistic fuzzy interaction partitioned Bonferroni mean operators and their application to multi-attribute group decision making. Inf. Sci. 2017, 411, 98-121. [CrossRef]

41. Liu, P.; Li, H. Interval-valued intuitionistic fuzzy power Bonferroni aggregation operators and their application to group decision making. Cogn. Comput. 2017, 9, 494-512. [CrossRef]

42. Liu, P.; Li, Y.; Antuchevičienè, J. Multi-criteria decision-making method based on intuitionistic trapezoidal fuzzy prioritized OWA operator. Technol. Econ. Dev. Econ. 2016, 22, 453-469. [CrossRef]

43. Xia, M.; Xu, Z. Hesitant fuzzy information aggregation in decision making. Int. J. Approx. Reason. 2011, 52, 395-407. [CrossRef]

44. Wang, J.Q.; Wu, J.T.; Wang, J.; Zhang, H.Y.; Chen, X.H. Interval-valued hesitant fuzzy linguistic sets and their applications in multi-criteria decision-making problems. Inf. Sci. 2014, 288, 55-72. [CrossRef]

45. Li, Y.; Liu, P.; Chen, Y. Some single valued neutrosophic number Heronian mean operators and their application in multiple attribute group decision making. Informatica 2016, 27, 85-110. [CrossRef]

46. He, J.K.; Teng, F.; Qi, Y. Towards a new climate economics: Research areas and prospects. Chin. J. Popul. Res. Environ. 2015, 13, 1-9. [CrossRef]

47. Jain, P. Population and development: Impacts on environmental performance. Chin. J. Popul. Res. Environ. 2016, 14, 208-214. [CrossRef]

48. Wu, H.X. The impact of climate changes on mass events in China. Chin. J. Popul. Resour. Environ. 2016, 14, 11-15. [CrossRef]

49. Zhang, X. A new approach to natural capital sustainable development. Chin. J. Popul. Res. Environ. 2016, 14, 105-111. [CrossRef] 